discussion. In so doing we have dug down to the very roots of geometry and dynamics but we have also touched the branches where the shoots are pushing out. Too much must not be claimed. Yet if the point-set theory is not the sap of the tree, it is at least one of its most indispensable ingredients. The usefulness of any mathematical theory must be determined not by its isolation but by its ability to combine with other theories. In this the point-set theory has shown itself most elastic. Had I more time I would attempt to show you that it offers many of the same advantages as analytical geometry. While strong for analysis and decomposition, it is equally strong on the constructive side. Complex groupings of points are made simple, and the way is thus prepared for new discovery. And above all, in its development the arithmetization of analysis is kept close to geometrical intuition.

In tracing the service of the theory of point-sets in geometry and dynamics, we have found only in part achievement, in part present evolution and promise. But it is precisely because of this mocking incompleteness that I have chosen for my topic today the rôle of the point-set theory in geometry and dynamics, trusting that for you also this will be its lure.

UNIVERSITY OF WISCONSIN, MADISON, Wis.

\title{
AN ENUMERATION OF INTEGRAL ALGEBRAIC POLYNOMIALS.
}

BY PROFESSOR A. B. FRIZELL.

(Read before the American Mathematical Society, January 1, 1915.)

THE proof given by Weber* that the algebraic numbers form a countable set orders them according to the values of a certain function of the coefficients in their defining equations. The present note suggests a more direct enumeration of these equations. The algebraic polynomials $\sum_{i=0}^{n} a_{i} x^{n-i}$ in which all coefficients are natural numbers can be put into one-to-one correspondence with the set of natural numbers by the fol-

\footnotetext{
* Algebra, Bd. II, p. 824.
} 
lowing device. To every $k x^{r}$ assign the number $p_{r}^{k}$, where $p_{0}=2$ and $p_{r}$ denotes the $r$ th odd prime when $r \neq 0$. To the sum $k_{1} x^{r_{1}}+k_{2} x^{r_{2}}$ assign the product $p_{r_{1}}^{k_{1}} \cdot p_{r_{2}}^{k_{2}}$, and so on. Thus the polynomial $x^{8}+3 x^{4}+2$ will have the number $p_{8} \cdot p_{4}^{3} \cdot p_{0}^{2}=23 \times 11^{3} \times 2^{2}=122,452$. Conversely, the polynomial numbered $360=5 \times 3^{2} \times 2^{3}=p_{2} \cdot p_{1}^{2} \cdot p_{0}^{3}$ will be $x^{2}+2 x+3$.

The polynomials in which negative integers occur as coefficients may be put into one-to-one correspondence with the set of positive fractions in their lowest terms by substituting division for multiplication in the above process. Thus to the polynomial $x^{8}-3 x^{4}+2$ will be assigned the fraction

$$
\frac{p_{8} \cdot p_{0}^{2}}{p_{4}^{3}}=\frac{92}{1331} \text {. }
$$

These fractions can be counted off diagonally from the wellknown rectangular array where every element in the $n$th row has the denominator $n+1$, and numbered with odd numbers, reserving the even numbers for the polynomials with positive coefficients, so that finally $x^{8}+3 x^{4}+2$ is to be numbered 244,904. For example, to identify number 605 $=2 \times 303-1$, we first obtain the greatest value of $n$ which makes

$$
\frac{n(n+1)}{2} \leqq 303 .
$$

This shows that number 303 in the rectangular array is the third fraction in the 25th diagonal, counting upwards. To avoid repetitions we will agree that the highest term in every polynomial shall be positive. This requires that the numerator of each fraction shall contain a prime factor greater than any in the denominator. Hence number 303 is

$$
\frac{11}{24}=\frac{p_{4}}{p_{1} \cdot p_{0}^{3}} \sim x^{4}-x-3 .
$$

To determine the number which belongs to $x^{8}-3 x^{4}+2$, we count in row 1,330 up to 92 , which is the 41st. Therefore $92 / 1331$ is in diagonal 1,370 . The sum of the first 1,369 diagonals is 937,765 and the required number is $1,875,611$.

McPherson, Kans. 